\title{
A series of unfortunate events
}

\section{How should a health system react after preventable medical errors?}

\author{
Jamie Riggs, Carlos Muzlera \\ Faculty Reviewer: Merrick Zwarenstein, MBBCh, Msc, PhD (Department of Family Medicine)
}

\section{CASE INTRODUCTION}

Mr. B presents to the ED with a 4 day history of dyspnea. He is a smoker, and was diagnosed one year ago with systolic heart failure (NYHA II). He has a history of hypertension, and is on enalapril 10mg PO BID and labetalol 200mg PO q12h. Physical exam reveals bilateral crackles and moderate peripheral edema. The ED physician orders a chest X-ray, and observes signs of pulmonary edema. A decision is made to admit Mr. B, but it proves difficult to diurese him, and the decision is made to insert a Foley catheter on the ward. On the third night of his stay, he complained to a member of the cleaning staff of severe pain in his right leg. The staff member subsequently notified the nurse, who was able to contact the resident on call. A bedside ultrasound was performed, and confirmed the presence of a DVT. The resident also noted that the patient had not been started on DVT prophylaxis. After morning rounds the patient was started on anticoagulation, and his pain resolved within a few hours. Now on his 4th day in hospital, the nurse noted that Mr. B was now febrile, and that he was producing cloudy urine. The catheter is removed and Mr. B is started on empiric antibiotic therapy, and a few days later the infection resolves. However, Mr. B spent 5 extra days in hospital and was discharged feeling extremely displeased with his care. You are the hospital director of quality improvement, and have been asked to review the case and suggest solutions.

\section{WHAT IS QUALITY IMPROVEMENT AND WHY SHOULD WE BOTHER?}

Quality improvement (QI) is defined as "the combined and unceasing efforts of everyone-healthcare professionals, patients and their families, researchers, payers, planners and educators, to make the changes that will lead to better patient outcomes (health), better system performance (care) and better professional development (learning)." ${ }^{1}$ The Institute for Healthcare Improvement, a leading QI organization based in the United States, advocates for the use of a systematic QI process, outlined in Figure 1. In order to create change, the problem must be rigorously defined, including the patient population and health systems that will be affected by the proposed interventions. Quantitative measures must be defined prior to implementing any changes in order to determine whether a specific change has improved the outcome of interest. Ideas for interventions should be solicited from healthcare professionals, others working in the system, and those who have previously completed successful changes. It is important to note that traditionally, QI has been distinguished from research by it's focus on local problems and it's use of Plan-Do-Study-Act cycles of rapid data collection and refinement of interventions. QI was also normally not carried out with the intention of publishing scholarly papers. ${ }^{2}$ This distinction is currently being blurred, with the advent of QI-focused journals, as well as QI work published in prestigious traditional research journals leading some to question whether QI projects should be subject to similar oversight as other research programs. ${ }^{3-6}$

Preventable complications in hospitalized patients represent an enormous burden on our healthcare system. The WHO has reported that $7 \%$ of hospitalized patients in developed countries will acquire a healthcare associated infection, with rates as high as $30 \%$ in patients in intensive care units. ${ }^{7}$ The Canadian Patient Safety Institute reports that about 8,000 Canadians die each year from hospital acquired infections, and that in 2010 \$129 million was spent treating these infections. ${ }^{8}$ A meta-analysis of hospitalized patients in Vancouver, BC, found that infection with vancomycin-resistant enterococci (VRE) increased the length of stay by an average of $68 \%$, or 13.8 days, and increased costs by $61.9 \%$ or $\$ 17,949$ in absolute terms. ${ }^{9}$ For these reasons, reducing hospital-acquired infection has been a major goal of quality improvement efforts. In the United States a program aimed at reducing central-line infections has been adopted in at least 45 states, contributing to a $63 \%$ decrease in these infections between 2001 and 2009. The program uses a model focused on collaboration both within and between states and is supported by centralized data collection and a distribution tool that provides each participating hospital with real-time reports of their infection rates. ${ }^{10}$

Although national level estimates of hospital acquired DVT are not available, Canadian data suggests that DVT also represents a serious cost to our system, with each case averaging $\$ 5180 .{ }^{11}$ At a large US teaching hospital, a QI effort based around provider education, reminders, decision support tools, data audits and feedback was implemented over a 3 year period to improve adherence to DVT prophylaxis guidelines. Over this period of time, adherence to the guidelines increased from $63 \%$ to $96 \%$, while rates of hospital acquired DVT fell from 2.6 per 1,000 discharges to 0.2 per 1,000 discharges. ${ }^{12}$ Clearly, avoiding preventable complications should be a goal for hospitals as well as our healthcare system, and well-conceived QI measures represent an important method for achieving this goal.

\section{EVALUATION OF THE CURRENT CASE}

What are the possible lapses that have contributed to Mr. B's complications? At multiple points throughout this case, breakdowns in communication contributed to the eventual outcomes. Evidence has shown that handover procedures are frequently in- 
complete, and that in some cases information considered important by physicians and nurses is omitted, ${ }^{13}$ and that ineffective communication is one of the leading causes of medical error. ${ }^{14}$

Although this case does not specify whether standard protocols for DVT prophylaxis or catheter insertions are used at this hospital, it is possible that both complications may have been avoided by the use of "bundled" care..$^{15}$ Electronic medical records often have these bundled orders that are implemented automatically upon the initiation of certain procedures.

It is important to recognize what went well during the course of Mr. B's stay in order to acknowledge and learn from these positive actions. Well trained housekeeping staff were able to quickly alert the nursing staff to Mr. B's problem, and he was subsequently able to get treatment quickly. Additionally, despite his complications, Mr. B was able to be discharged to home with no long term consequences. Finally, the fact that the hospital initiated a QI effort is a crucial step towards creating positive change in this system.

\section{STRATEGIES FOR IMPROVEMENT}

A first step for initiating any QI initiative is to involve the right people in the effort. This team includes members with expertise in the technical details of the case, a day-to-day leader of the initiative, representation from allied healthcare professions affected by the proposed changes, as well as a sponsor or champion in hospital management to advocate for the project. ${ }^{16}$ Once established, this QI team should actively solicit ideas for improvement initiatives, some of which are detailed below. Before undertaking any changes, quantitative outcome measures must be defined in order to track the success of the intervention. These measures will vary based on the aspect of care being targeted, but must be relatively easily tracked over time and must be meaningful in the context of the goals of the QI project. Potential strategies for change in this case include:

Standardized communications: The Situation, Background, Assessment, Recommendation (SBAR) method has been proposed as a method to ensure that all team members are on the same page. ${ }^{14}$ Using this method, all members of the healthcare team frame their communications around four questions: What is going on with the patient? What is the clinical background or context? What do I think the problem is? What do I think needs to be done for the patient?

Team huddles: Implementing focused 'huddles' with all members of the care team at the beginning of every shift in order to set out the goals and action items to be attended to has been shown to reduce the need for interruptions in order to seek clarification later in the shift. ${ }^{17}$

Goal oriented multi-disciplinary rounds: Daily rounds including all members of the healthcare team are focused using a patient centered approach, where the aim is to ensure that all members of the team are clear on what the goals of care are for that day. ${ }^{17}$ Evidence suggests that these rounds can significantly increase the proportion of nurses and physicians who understand the goals of care for that day, and can reduce the length of stays in the ICU by up to $50 \% .^{3}$

Bundles of Care: Bundles are sets of evidence-based practices designed to prevent complications. Bundles may be designed by the QI team, in collaboration with other content experts, or taken from existing literature. In one study, implementation of care bundles along with multi-disciplinary rounds resulted in significant reductions in nosocomial UTIs, including four consecutive months without a catheter associated UTI in an ICU setting. ${ }^{15}$

You form a working group consisting of the unit charge nurse, an infection control expert, a consultant internist on the same unit and yourself as hospital administration champion. Over the course of three meetings, the team reviewed the case and identified areas for improvement. Initially, two measures were selected: the rate of catheter associated infections on the unit, and nursing staff's self-reported knowledge of the assessment and treatment plan for patients under their care. After baseline data was collected, the team implements two changes. First, goal oriented multi-disciplinary rounds will occur on a daily basis. Additionally, an evidence based bundle of care is put in place through the hospitals electronic medical record for patients receiving urinary catheters. Data collection will continue throughout the trial period, lead by the unit charge nurse, and both interventions will be re-evaluated after one month.
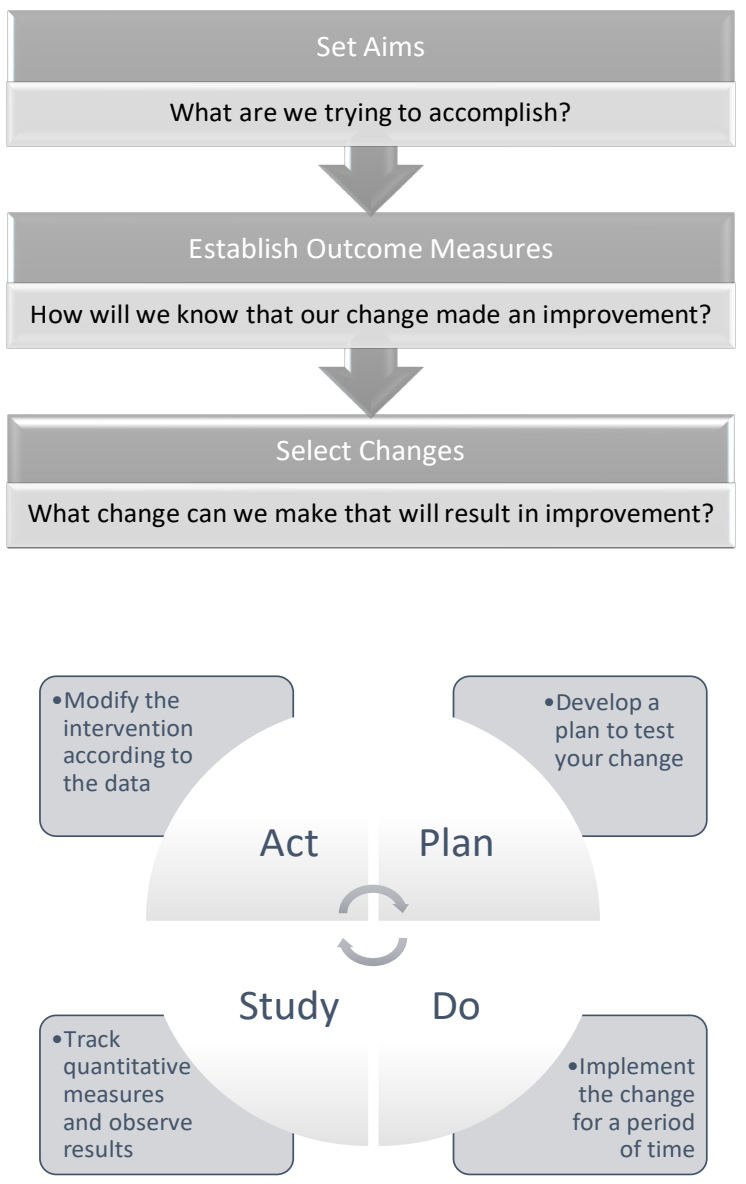

Figure 1. The systematic process of Quality Improvement. Adapted from the Institute for Healthcare Improvement's Model for Improvement ${ }^{18}$ 


\section{CONCLUSION}

This case has illustrated only a few of the nearly limitless opportunities for change in healthcare. QI, as a systematic cycle of iterative changes based on locally generated data, is a template that may be applied in many other healthcare settings. By using a systematic approach, we are better able to evaluate changes that have the potential to tackle the many challenges facing our healthcare systems and allow others to learn from our work.

\section{REFERENCES}

1. Batalden PB, Davidoff F. What is "quality improvement" and how can it transform healthcare? Qual Saf Healthc. 2007;16:2-3.

2. Bellin E, Dubler NN. The Quality Improvement - Research Divide and the Need for External Oversight. Am J Public Health. 2001;91(9):15127.

3. Pronovost P, Berenholtz S, Dorman T, et al. Improving communication in the ICU using daily goals. J Crit Care. 2003 Jun;18(2):71-5.

4. Palevsky PM, Washington MS, Stevenson, et al. Improving compliance with the dialysis prescription as a strategy to increase the delivered dose of hemodialysis: an ESRD Network 4 quality improvement project. Adv Ren Replace Ther. 2000 Oct;7(4 Suppl 1):S21-30.

5. Cretin S, Keeler EB, Lynn J, et al. Should patients in quality-improvement activities have the same protections as participants in research studies? JAMA. 2000 Oct 11;284(14):1786; author reply 1787-89.

6. Lynn J. When does quality improvement count as research? Human subject protection and theories of knowledge. Qual Saf Health Care. $2004 \mathrm{Feb} ; 13(1): 67-70$.

7. WHO. Health care-associated infections: Fact Sheet. 2014. Available from: www.who.int/gpsc/country_work/gpsc_ccisc_fact_sheet_en.pdf.

8. CPSI. Healthcare Associated Infections [Internet]. 2016 [cited 2017 Feb 18]. Available from: http://www.patientsafetyinstitute.ca/en/Topic/Pages/Healthcare-Associated-Infections-(HAI).aspx.

9. Lloyd-Smith P, Younger J, Lloyd-Smith E, et al. Economic analysis of vancomycin-resistant enterococci at a Canadian hospital: assessing attributable cost and length of stay. J Hosp Infect. 2013 Sep;85(1):54-9.

10. Provonost P, Marsteller J, Goeschel, C. Preventing Bloodstream Infections: A Measurable National Success Story in Quality Improvement. Health Aff. 2011 Apr;30(4):628-34.

11. Guanella R, Ducruet T, Johri M, et al. Economic burden and cost determinants of deep vein thrombosis during 2 years following diagnosis: a prospective evaluation. J Thromb Haemost. 2011 Dec;9(12):2397405.

12. Bullock-Palmer RP, Weiss S, Hyman C. Innovative approaches to increase deep vein thrombosis prophylaxis rate resulting in a decrease in hospital-acquired deep vein thrombosis at a tertiary-care teaching hospital. J Hosp Med. 2008 Mar;3(2):148-55.

13. Siddiqui N, Arzola C, Iqbal M, et al. Deficits in information transfer between anaesthesiologist and postanaesthesia care unit staff: an analysis of patient handover. Eur J Anaesthesiol.2012 Sep;29(9):438-45.

14. Leonard M, Graham S, Bonacum D. The human factor: the critical importance of effective teamwork and communication in providing safe care. Qual Saf Health Care. 2004 Oct;13 Suppl 1:i85-90.

15. Jain M, Miller L, King D, et al. Decline in ICU adverse events, nosocomial infections and cost through a quality improvement initiative focusing on teamwork and culture change. Qual Saf Heal Care. 2006;(15):235-9.

16. IHI. Science of Improvment: Forming the Team [Internet]. 2017.
Available from: http://www.ihi.org/resources/Pages/HowtoImprove/ ScienceofImprovementFormingtheTeam.aspx.

17. Dingley C, Daugherty K, Derieg MK, et al. Improving Patient Safety Through Provider Communication Strategy Enhancements. In: Henriksen K, Battles J, Keyes M, editors. Advances in Patient Safety: New Directions and Alternative Approaches (Vol 3: Performance and Tools) [Internet]. Rockville, MD: Agency for Healthcare Research and Quality (US); 2008. p. 1-18. Available from: https://www.ncbi.nlm.nih.gov/ books/NBK43663/.

18. IHI. Science of Improvement: How to Improve [Internet]. [cited 2017 Feb 17]. Available from:http://www.ihi.org/resources/Pages/HowtoImprove/ScienceofImprovementHowtoImprove.aspx. 